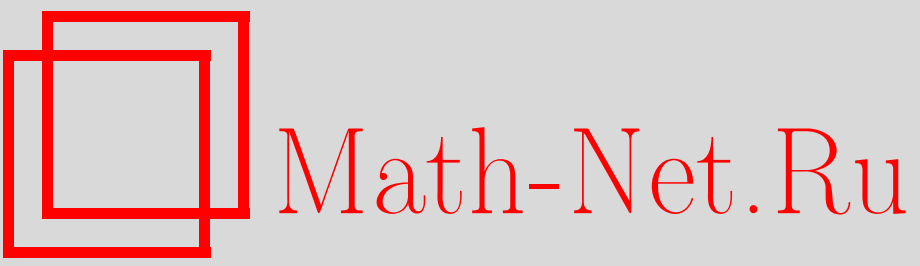

Л. П. Гинзбург, Проблема локализации в одномерных неупорядоченных системах (новый подход), ТМФ, 1996, том 106, номер 3, 425-437

DOI: https://doi.org/10.4213/tmf1127

Использование Общероссийского математического портала Math-Net.Ru подразумевает, что вы прочитали и согласны с пользовательским соглашением

http://www. mathnet.ru/rus/agreement

Параметры загрузки:

IP : 54.174 .149 .18

26 апреля 2023 г., 17:41:34 
ТЕОРЕТИЧЕСКАЯ

И МАТЕМАТИЧЕСКАЯ

ФИЗИКА

Том 106, № 3

март, 1996

\section{Л.П. Гинзбург \\ ПРОБЛЕМА ЛОКАЛИЗАЦИИ В ОДНОМЕРНЫХ НЕУПОРЯДОЧЕННЫХ СИСТЕМАХ (НОВЫЙ ПОДХОД)}

\footnotetext{
Доказанная в 1951 г. теорема Молчанова [15] применяется к изучению проблемы локализации одноэлектронных состояний в одномерных неупорядоченных системах. Теорема позволяет подойти к проблеме с общих позиций и устанавливает новый критерий локализации, основанный на асимптотических свойствах случайного потенциала. Показано, что в условиях диагонального беспорядка теорема не приводит к новым результатам: все состояния оказываются локализованными. Однако в условиях структурного беспорядка из теоремы следует, что при сравнительно слабых ограничениях все состояния могут оказаться делокализованными.
}

\section{ВВЕДЕНИЕ}

Возможно, в работе [1] впервые было сформулировано положение, согласно которому в одномерных (1D) неупорядоченных системах, описываемых одноэлектронным уравнением Шредингера, все состояния должны быть локализованы. Это утверждение, с тех пор исследованное и повторенное во многих работах (см., например, [2-6]), к настоящему времени приобрело характер всеобшего убеждения, причем нередко безоговорочного. Но оговорки сушествуют и, на наш взгляд, серьезные. Например, в ряде работ ([7-10] и др.) показано, что в условиях структурной неупорядоченности $(\mathrm{CH})$ возможно появление состояний, которые делокализованы. Более того, например, в работе [11] был продемонстрирован ряд CH1D-систем, которые вообше ведут себя, как хорошие проводники.

В связи со сказанным у автора сложилось мнение, что существующие на сегодняшний день методы анализа проблемы локализации одноэлектронных состояний в 1D-системах не обладают достаточной обшностью и что поиск более общего подхода крайне необходим. Исходные принципы такого подхода известны. В работе [12] показано, что для правильной оценки характера одноэлектронного состояния (т.е. выяснения, локализовано оно или нет) необходим переход к бесконечной области. При этом однозначным признаком локализации состояний является дискретность энергетического спектра, а признаком делокализации - его непрерывность. Этот вопрос изучался в работе [13], где показано, что спектр бесконечной одномерной неупорядоченной системы является чисто дискретным. Однако сушественным пунктом доказательства является свойство марковости случайного потенциала $V(x)$, который входит в уравнение Шре- 
дингера

$$
-\frac{\hbar^{2}}{2 m} \frac{d^{2} \varphi(x)}{d x^{2}}+V(x) \varphi(x)=E \varphi(x) .
$$

Это свойство заключается в том, что любые функционалы от $V(x)$, рассматриваемые слева и справа от некоторой точки на бесконечной прямой, должны быть статистически независимы. Ясно, что такое условие может реализоваться, только если вклад в интегралы переноса вносит малое число ближайших соседей (атомов), а в наиболее чистом виде - просто пара ближайших соседей. Парадокс состоит в том, что, как показано в [14], в условиях СН именно учет ближайших соседей может оказаться причиной сушественного искажения вида энергетического спектра. Поэтому несмотря на мощный аппарат доказательства, примененный в [13], вывод об обязательной локализации состояний 1D неупорядоченных систем не представляется достаточно общим.

В связи с изложенным автор воспользовался другим подходом к проблеме дискретности спектра 1D-систем. Речь идет о теореме Молчанова (TM), доказанной еше в 1951 г. [15], согласно которой в условиях уравнения (1), заданного на неограниченной прямой, необходимым и достаточным условием дискретности спектра является равенство

$$
\lim _{Z \rightarrow \pm \infty} \int_{Z-w}^{Z} V(x) d x=\infty,
$$

где $w$ - произвольная, но конечная длина. При этом единственное ограничение, которое накладывается на функцию $V(x)$, сводится к соотношению

$$
V(x)>-\infty .
$$

На наш взгляд, привлекательность ТМ состоит не только в общности, но и в известной простоте предлагаемого критерия, под который подпадают как случайные, так и детерминированные потенциалы. Во всяком случае условие (3) представляется гораздо более слабым, нежели требование марковости, а из (2) следует, что о локализации состояния можно судить, изучая только асимптотические свойства $V(x)$.

Разумеется, не всегда очевидно, как к той или иной системе можно применить критерий (2), (3). Вполне возможно, что в конкретных случаях более удобными могут оказаться уже известные методы. Однако представляется странным, что до сих пор никто из исследователей проблемы локализации в $1 \mathrm{D}$ неупорядоченных системах не обратил внимания на сушествование такого обшего критерия, как TM, и не попытался хотя бы сравнить с ней полученные результаты ${ }^{1)}$.

Цель настояшей статьи состоит в попытке восполнить этот пробел и, опираясь на $\mathrm{TM}$, проанализировать проблему 1D-локализации в условиях по позвожности обшего вида потенциала $V(x)$. В следующем разделе рассматривается случай диагонального беспорядка, где

$$
V(x)=\sum_{n} A_{n} W(x-a n) .
$$

Здесь $n=1,2,3, \ldots, a=$ const, а множители $A_{n}$ - случайные, некоррелированные величины, которые ограничены только условием: $\left|A_{n}\right|<\infty$. Что же касается функции

\footnotetext{
1) Это тем более странно, что до настоящего времени ТМ широко цитируется в математической литературе (см., например, [16]).
} 
$W(x)$, то мы будем предполагать, что она интегрируема и удовлетворяет следующим не слишком жестким условиям. Во-первых,

$$
W(x)=W(-x)
$$

Во-вторых, при $|x| \rightarrow \infty$ функция $W(x)$ достаточно быстро стремится к нулю. Быстрота эта определяется тем, что при любых значениях переменных $p$ и $q$ и любой непрерывной конечной функции $A(x)$ всегда найдется такая конечная длина $S_{0} \gg a$, что с любой требуемой точностью

$$
\int_{-\infty}^{\infty} d x W(x) A(x+p) e^{i(x+p) q} \simeq \int_{-S_{0}}^{S_{0}} d x W(x) A(x+p) e^{i(x+p) q}
$$

Наконец, мы будем считать, что фурье-образ функции $W(x)$

$$
F(q)=\int_{-\infty}^{\infty} d x W(x) e^{-i q x} \simeq \int_{-S_{0}}^{S_{0}} d x W(x) e^{-i q x}
$$

может содержать в верхней полуплоскости только конечное число особенностей в виде полюсов

Заметим, что часто рассматриваемая модель случайного сплава [3, 17-19], где

$$
V(x)=\sum_{n} A_{n} \delta(x-a n)
$$

является частным случаем [4].

Полученный в следующем разделе результат не противоречит тому, что ожидалось: все состояния, описываемые потенциалом (4), оказываются локализованными. Однако автор не уверен, что методы, применявшиеся в работах [3, 17-19], позволили бы также сравнительно легко этот результат получить, если от частного случая (8) перейти к более общей модели (4).

В разделе 2 рассмотрен типичный случай $\mathrm{CH}$-системы, когда потенциал определяется соотношением

$$
V(x)=\sum_{n} U\left(x-X_{n}\right)
$$

В выражении (9) координаты $X_{n}$ являются случайными и полностью некоррелированными величинами. Здесь показано, что при сравнительно слабых ограничениях, накладываемых на $U(x)$ (и при соблюдении (3)), все состояния могут оказаться делокализованными.

Результат, полученный в разделе 2, противоречит выводам ряда работ (в частности, [13]). В последнем разделе в предварительном порядке обсуждается возможная причина расхождения. 


\section{1. ДИАГОНАЛЬНЫЙ БЕСПОРЯДОК}

В условиях (4) потенциал $V(x)$ определяется набором одинаковых потенциалов $W(x-a n)$, каждый из которых входит в (4) со случайным весом $A_{n}$. В этих условиях образец длины

$$
L=a G,
$$

где $G$ - сколь угодно большое целое число, можно считать репрезентативным. При этом, очевидно, можно подразумевать, что, в частности,

$$
a G \gg a+w+S_{0} .
$$

Учитывая (11), можно написать

$$
\int_{a G-w}^{a G} V(x) d x=\int_{-w}^{0} V(\alpha+a G) d \alpha \simeq w V(a G) .
$$

Введем непрерывную функцию $A(x)$, которая подчинена единственному условию

$$
A(a n)=A_{n}
$$

В этом случае, принимая во внимание (4), (5), (10), (11), будем иметь

$$
V(a G)=\sum_{n=1}^{G} \int_{-\infty}^{\infty} d z \delta(a n-a G-z) A(z+a G) W(z) .
$$

Заменим $\delta$-функцию выражением

$$
\delta(a n-a G-z)=\frac{1}{2 \pi} \int_{-\infty}^{\infty} d q \exp \left(-\frac{a}{G}|q|\right) e^{i(a n-a G-z) q}
$$

и примем во внимание (6), (11). В этом случае

$$
V(a G)=\frac{A(a G)}{2 \pi} \int_{-S_{0}}^{S_{0}} W(z) d z \int_{-\infty}^{\infty} d q \exp \left(-\frac{a}{G}|q|\right) e^{-i(a G+z) q} \sum_{n=1}^{G} e^{i a n q}
$$

Сумма в правой части (16) представляет собой геометрическую прогрессию. Поэтому

$$
\sum_{n=1}^{G} e^{i a n q}=e^{i a q} \frac{1-e^{i a G q}}{1-e^{i a q}}
$$

Подставив (17) в (16) и снова учитывая (11), мы сможем написать

$$
V(a G)=\frac{1}{2 \pi}\left(I_{1}-I_{2}\right)
$$




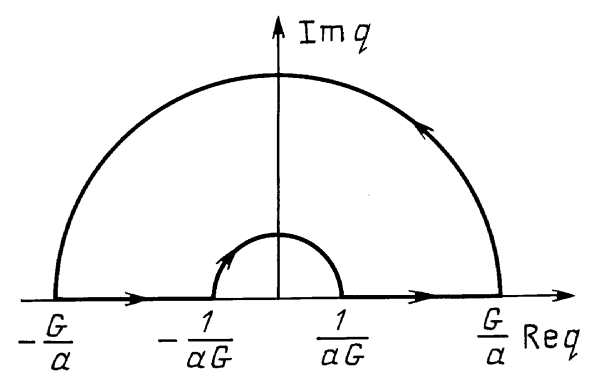

где

$$
\begin{aligned}
& I_{1}=A(a G) \int_{-S_{0}}^{S_{0}} W(z) d z \int_{-\infty}^{\infty} d q e^{-\frac{a}{G}|q|} \frac{e^{-i a G q}}{1-e^{i a q}}, \\
& I_{2}=A(a G) \int_{-S_{0}}^{S_{0}} W(z) d z \int_{-\infty}^{\infty} d q e^{-\frac{a}{G}|q|} \frac{e^{-i z q}}{e^{-i a q}-1} .
\end{aligned}
$$

Для вычисления $I_{1}$ преобразуем внутренний интеграл к виду

$$
\begin{aligned}
\int_{-\infty}^{\infty} d q e^{-\frac{a}{G}|q|} \frac{e^{-i a G q}}{1-e^{i a q}}= & \int_{-\infty}^{\infty} d q e^{-\frac{a}{G}|q|} \frac{e^{-i a G q}-1}{e^{-i a q}-1} e^{-i a q}+ \\
& +\int_{-\infty}^{\infty} d q e^{-\frac{a}{G}|q|} \frac{1}{1-e^{i a q}}
\end{aligned}
$$

Снова применяя правило сложения геометрической прогрессии, будем иметь

$$
\frac{e^{-i a G q}-1}{e^{-i a q}-1} e^{-i a q}=\sum_{n=1}^{G} e^{-i a n q}
$$

где $n=1,2,3, \ldots$. Подставив $(22)$ в первый интеграл правой части $(21)$, получим

$$
\begin{gathered}
\int_{-\infty}^{\infty} d q e^{-\frac{a}{G}|q|} \frac{e^{-i a G q}-1}{e^{-i a q}-1} e^{-i a q}=\int_{-\infty}^{\infty} d q e^{-\frac{a}{G}|q|} \sum_{n=1}^{G} e^{-i a n q}= \\
=\sum_{n=1}^{G} \int_{-\infty}^{\infty} d q e^{-\frac{a}{G}|q|} e^{-i a n q}=2 \pi \sum_{n=1}^{G} \delta(\text { an })=0
\end{gathered}
$$

Далее, используем контур, изображенный на рисунке. Учитывая, что по условию число $G$ сколь угодно велико, можем написать

$$
\begin{aligned}
\int_{-\infty}^{\infty} d q e^{-\frac{a}{G}|q|} \frac{1}{1-e^{i a q}} \simeq & -i \frac{G}{a} \int_{0}^{\pi} d \varphi e^{i \varphi-1} \frac{1}{1-e^{i G \cos \varphi e^{-G \sin \varphi}}-} \\
& -\frac{i}{a G} \int_{\pi}^{0} d \varphi \exp \left[i \varphi-\left(\frac{1}{G}\right)^{2}\right] \frac{1}{1-\exp \left(\frac{i}{G} e^{i \varphi}\right)}
\end{aligned}
$$


При достаточно больших $G$ с точностью до сколь угодно малых окрестностей точек $\varphi=0$ и $\varphi=\pi$ имеем $\exp (-G \sin \varphi) \ll 1$. Поэтому из $(24)$ следует

$$
\int_{-\infty}^{\infty} d q e^{-\frac{a}{G}|q|} \frac{1}{1-e^{i a q}} \simeq \frac{1}{a}\left(2 G e^{-1}-\pi\right)
$$

Отсюда, подставляя (21), (23), (25) в (19), получим

$$
I_{1} \simeq \frac{A(a G)}{a}\left(2 G e^{-1}-\pi\right) \int_{-S_{0}}^{S_{0}} W(z) d z .
$$

Для вычисления интеграла $I_{2}$ учтем, что на основании $(20),(7)$ его можно представить в виде

$$
I_{2}=A(a G) \int_{-\infty}^{\infty} d q e^{-\frac{a}{G}|q|} \frac{F(q)}{e^{-i a q}-1}
$$

Согласно (7)

$$
F(0)=\int_{-\infty}^{\infty} W(x) d x \simeq \int_{-S_{0}}^{S_{0}} W(x) d x .
$$

Поэтому особенности функции

$$
\Phi(q)=e^{-\frac{a}{G}|q|} \frac{F(q)}{e^{-i a q}-1}
$$

стоящей под знаком интеграла в $(27)$, появляются в точке $q=0$, а также в точках $q=b_{i}$, которые являются полюсами функции $F(q)$, расположенными в верхней полуплоскости. Отсюда, снова используя контур на рисунке, будем иметь

$$
\begin{aligned}
I_{2} & \simeq-i \frac{A(a G)}{a G} \int_{\pi}^{0} d \varphi \exp \left[i \varphi-\left(\frac{1}{G}\right)^{2}\right] \frac{F\left(\frac{1}{a G} e^{i \varphi}\right)}{\exp \left(-i \frac{1}{G} e^{i \varphi}\right)-1}+2 \pi i A(a G) \sum_{i=1}^{N_{0}} \operatorname{res} \Phi\left(b_{i}\right) \simeq \\
& \simeq-\frac{\pi}{a} A(a G) \int_{-S_{0}}^{S_{0}} W(x) d x+2 \pi i A(a G) \sum_{i=1}^{N_{0}} \operatorname{res} \Phi\left(b_{i}\right)
\end{aligned}
$$

где $N_{0}$ - количество полюсов $F(q)$ в верхней полуплоскости и учтено (29). Таким образом, подставив (26), (30) в (18), получим

$$
V(a G) \simeq \frac{A(a G)}{2 \pi}\left(\frac{2 G e^{-1}}{a} \int_{-S_{0}}^{S_{0}} W(z) d z-4 \pi^{2} i \sum_{i=1}^{N_{0}} \operatorname{res} \Phi\left(b_{i}\right)\right)
$$

Отсюда, приняв во внимание (12), а также то, что по условию $N_{0}$ конечно, можем написать

$$
\lim _{G \rightarrow \infty} \int_{a G-w}^{a G} V(x) d x=\lim _{G \rightarrow \infty} \frac{w}{\pi a} A(a G) G e^{-1} \int_{-S_{0}}^{S_{0}} W(z) d z .
$$

Из (32) видно, что если

$$
\lim _{G \rightarrow \infty} A(a G) \neq 0
$$


то правая часть (32) обрашается в бесконечность. Согласно ТМ последнее означает, что спектр системы дискретен и состояния локализованы.

Убедиться в справедливости (33) несложно. Если допустить, что

$$
\lim _{G \rightarrow \infty} A(a G)=0,
$$

то это означало бы, что для любого числа $0<\varepsilon \ll 1$ можно подобрать такую величину $G_{0}(\varepsilon)$, что при $G \geq G_{0}(\varepsilon)$

$$
|A(a G)| \leq \varepsilon .
$$

Но (35) означает, что для бесконечного множества значений $n \geq G_{0}(\varepsilon)$ величины $A(a n)=A_{n}$ должны быть малы. Последнее противоречит условию случайности коэффицциентов $A_{n}$ в выражении (4).

\section{2. СТРУКТУРНЫЙ БЕСПОРЯДОК}

Справедливо следуюшее

УТВЕРЖДЕНИЕ. Для того чтобы в условиях (9) (со случайными и некоррелированными координатами $X_{n}$ ) выполнялось вытекающее из ТМ условие делокализации

$$
\lim _{Z \rightarrow \infty} \int_{Z-w}^{Z} V(x) d x=C(w)<\infty,
$$

необходимо, чтобы в разложсении Фурье

$$
U\left(x-X_{n}\right)=\frac{1}{2 \pi} \int_{-\infty}^{\infty} R(q) e^{i\left(x-X_{n}\right) q} d q
$$

интеграл при любых $x$ сходился равномерно относительно $X_{n}$. При этом если функция $U(x)$ интегрируема и при больших $|x|$ выполняются соотношения типа

$$
U(x) \sim \exp \left(-\beta|x|^{n}\right), \quad|x|^{-n},
$$

где $n \geq 1$ и $\beta=$ const, то отмеченное условие равномерной сходимости является также достаточным.

Перед тем как приступить к доказательству, заметим, что уравнение (1) мы можем разделить на некоторую постоянную величину $V_{0}$, имеющую размерность энергии, и ввести "массу" $\tilde{m}=V_{0} a^{2} m$, где $a-$ постоянная, имеющая размерность длины. Поэтому без нарушения общности будем в дальнейшем рассматривать энергию и длину как безразмерные величины.

2.1. Доказательство необходимости. 1. Сформулируем условие, достаточное для равномерной сходимости интеграла в (37): существует такая длина $Z_{0}$, что при любых $Z \geq Z_{0}$ и $w>0$ величины вида

$$
D_{n}(Z, w)=\int_{Z-w}^{Z} U\left(x-X_{n}\right) d x
$$


остаются конечными. Такое условие важно, поскольку предполагается предельный переход $Z \rightarrow \infty$ и априори неясно, как при этом ведут себя величины, определяемые (39). Предположим, что $X_{m}>X_{n}$, и рассмотрим величину

$$
D_{m}(Z, w)=\int_{Z-w}^{Z} U\left(x-X_{m}\right) d x
$$

Сделаем замену: $x=\alpha+Z$. Кроме того, в (39) положим $Z=Z_{0}$, а в (40)-Z= $Z_{0}+$ $X_{m}-X_{n}$. Легко проверить, что

$$
\int_{-w}^{0} U\left(\alpha+Z_{0}-X_{n}\right) d \alpha=D_{n}\left(Z_{0}, w\right)=D_{m}\left(Z_{0}+X_{m}-X_{n}, w\right) .
$$

Сходимость интеграла в (37) (при любых $Z$ в (36)) означает, что для любого $w>0$ и любого числа $0<\varepsilon \ll 1$ сушествует такая величина $K_{n}(Z ; w, \varepsilon)$, что при любых $K \geq K_{n}(Z ; w, \varepsilon)$ справедливо равенство

$$
U\left(x-X_{n}\right)=\frac{1}{2 \pi} \int_{-K}^{K} R(q) e^{i\left(x-X_{n}\right) q} d q+\eta_{K}\left(x-X_{n}\right),
$$

где

$$
\left|\eta_{K}\left(x-X_{n}\right)\right| \leq \frac{\varepsilon}{2 w}
$$

Аналогичным образом можно указать величину $K_{m}(Z ; w, \varepsilon)$ такую, что при любых $K^{\prime} \geq K_{m}(Z ; w, \varepsilon)$

$$
U\left(x-X_{m}\right)=\frac{1}{2 \pi} \int_{-K^{\prime}}^{K^{\prime}} R(q) e^{i\left(x-X_{m}\right) q} d q+\eta_{K^{\prime}}\left(x-X_{m}\right),
$$

где

$$
\left|\eta_{K^{\prime}}\left(x-X_{m}\right)\right| \leq \frac{\varepsilon}{2 w}
$$

Подставив (42), (44) соответственно в (39), (40) и в обоих случаях сделав замену $x=$ $\alpha+Z$, мы сможем написать

$$
\begin{aligned}
& \frac{1}{2 \pi} \int_{-w}^{0} d \alpha\left(\int_{-K}^{K} R(q) e^{i\left(\alpha+Z-X_{n}\right) q} d q\right)=D_{n}(Z, w)-\int_{-w}^{0} d \alpha \eta_{K}\left(\alpha+Z-X_{n}\right) \\
& \frac{1}{2 \pi} \int_{-w}^{0} d \alpha\left(\int_{-K^{\prime}}^{K^{\prime}} R(q) e^{i\left(\alpha+Z-X_{m}\right) q} d q\right)=D_{m}(Z, w)-\int_{-w}^{0} d \alpha \eta_{K^{\prime}}\left(\alpha+Z-X_{m}\right)
\end{aligned}
$$

Предположим теперь, что в (46) $Z=Z_{0}$, а в (47) $Z=Z_{0}+X_{m}-X_{N}$. Тогда, вычитая из первого равенства второе и приняв во внимание (41), получим

$$
\begin{gathered}
\frac{1}{2 \pi}\left|\int_{-w}^{0} d \alpha\left(\int_{-K}^{K} R(q) e^{i\left(\alpha+Z_{0}-X_{n}\right) q} d q-\int_{-K^{\prime}}^{K^{\prime}} R(q) e^{i\left(\alpha+Z_{0}-X_{n}\right) q} d q\right)\right|= \\
=\left|\int_{-w}^{0} d \alpha\left(\eta_{K^{\prime}}\left(\alpha+Z_{0}-X_{n}\right)-\eta_{K}\left(\alpha+Z_{0}-X_{n}\right)\right)\right| \leq \varepsilon,
\end{gathered}
$$


где неравенство следует из (43), (45). Так как величина $\varepsilon$ может быть сделана сколь угодно малой, а $w$ произвольно, то из (48) вытекает $K=K^{\prime}$, что означает $K_{n}(Z ; w, \varepsilon)=$ $K_{m}(Z ; w, \varepsilon)$. Учитывая, что координаты $X_{n}$ и $X_{m}$ выбраны нами произвольно, тем самым убеждаемся в равномерной сходимости интеграла (37) относительно $X_{n}$.

2. Покажем, что в условиях (36) величина $Z_{0}$, обладающая нужными свойствами, сушествует. Условие (36) означает, что для любого числа $0<\varepsilon \ll 1$ сушествует такая величина $Z_{0}(\varepsilon)$, что при любых $Z \geq Z_{0}(\varepsilon)$

$$
\int_{Z-w}^{Z} V(x) d x=\int_{Z-w}^{Z} \sum_{n} U\left(x-X_{n}\right)=C(w)+\Delta_{Z}(w),
$$

где

$$
\left|\Delta_{Z}(w)\right| \leqslant \varepsilon
$$

Из (39), (49) следует

$$
\sum_{n} D_{n}(Z, w)=C(w)+\Delta_{Z}(w) .
$$

Согласно (36) $C(w)$ - конечная величина, а добавок $\Delta_{Z}(w)$ в соответствии с (50) может быть сколь угодно малым. Отсюда следует, что величины $D_{n}(Z, w)$ в (51) тоже должны быть конечными. В противном случае между отдельными слагаемыми левой части (51) возникла бы корреляция, а это согласно (39) означало бы корреляцию между координатами $X_{n}$, что противоречит условию задачи.

Таким образом, при соблюдении (36) интеграл (37) при любых $x$ сходится относительно $X_{n}$ равномерно.

2.2. Доказательство достаточности. Рассмотрим образец длины $Z$ такой, что

$$
N Z \gg 1,
$$

где $N$ - средняя плотность координат $X_{n}$. Рассмотрим выражение

$$
\int_{Z-w}^{Z} V(x) d x=\int_{Z-w}^{Z} d x \sum_{n} \int_{-\infty}^{\infty} d q R(q) e^{i\left(x-X_{n}\right) q},
$$

где использовано разложение (37). Если интеграл по $q$ в (37), (53) сходится относительно $X_{n}$ равномерно, то процедуры суммирования и интегрирования в (53) можно поменять местами. В результате получим

$$
\sum_{n} \int_{-\infty}^{\infty} d q R(q) e^{i\left(x-X_{n}\right) q}=\int_{-\infty}^{\infty} d q R(q) P(q) e^{i x q},
$$

где

$$
P(q)=\sum_{n} e^{-i X_{n} q} .
$$

Величина $P(q)$ является суммой случайных некоррелированных величин. Поэтому ее следует оценивать вероятностными методами (тем более, что для нас достаточна порядковая оценка). Мы воспользуемся приемом, который был применен в работе [20] (см. также [21]). Согласно [20]

$$
P(q) \sim N Z \delta_{q, 0} \pm(N Z)^{1 / 2}\left(1-\delta_{q, 0}\right) .
$$


Из (56), (54), (53) следует

$$
\int_{Z-w}^{Z} V(x) d x \sim L_{1}+L_{2}
$$

где

$$
\begin{aligned}
& L_{1}=N \int_{Z-w}^{Z} d x \int_{-\infty}^{\infty} d q R(q) \frac{Z \delta_{q, 0}}{2 \pi}\left(1 \mp \frac{1}{(N Z)^{1 / 2}}\right) e^{i x q}, \\
& L_{2}= \pm \frac{(N Z)^{1 / 2}}{2 \pi} \int_{Z-w}^{Z} d x \int_{-\infty}^{\infty} d q R(q) e^{i x q} .
\end{aligned}
$$

Переходя к пределу $Z \rightarrow \infty$, мы можем в (58) положить

$$
\frac{Z}{2 \pi} \delta_{q, 0}=\delta(q)
$$

Отсюда

$$
\lim _{Z \rightarrow \infty} L_{1}=N w R(0)=N w \int_{-\infty}^{\infty} U(x) d x
$$

Положив в (59) $x=\alpha+Z$ и учитывая (37), будем иметь

$$
L_{2}= \pm \frac{(N Z)^{1 / 2}}{2 \pi} \int_{-w}^{0} d \alpha \int_{-\infty}^{\infty} d q R(q) e^{i(\alpha+Z) q}= \pm(N Z)^{1 / 2} \int_{-w}^{0} d \alpha U(\alpha+Z)
$$

Если выполняются условия типа (38), то при $Z \rightarrow \infty$

$$
U(\alpha+Z) \sim \exp \left(-\beta Z^{n}\right)
$$

или

$$
U(\alpha+Z) \sim Z^{-n}
$$

Подставив (63) или (64) в (62), легко убедиться в том, что

$$
\lim _{Z \rightarrow \infty} L_{2}=0
$$

Таким образом, комбинируя (57), (61), (65), получаем

$$
\lim _{Z \rightarrow \infty} \int_{Z-w}^{Z} V(x) d x \sim N w \int_{-\infty}^{\infty} U(x) d x
$$

Согласно ТМ соотношение (66) означает делокализацию электронных состояний. 


\section{3. ОБСУЖДЕНИЕ}

Для полноты картины ТМ была применена автором также к случаю периодического потенциала. Как и следовало ожидать, спектр оказался непрерывным. Таким образом, подводя итог, можно сказать, что в условиях периодического или диагонально неупорядоченного потенциала ТМ подтверждает уже известные факты: в первом случае состояния делокализованы, а во втором - локализованы. Однако результат предыдушего раздела может казаться неожиданным: при сравнительно слабых ограничениях состояния типичной CH1D-системы оказываются делокализованными. Насколько автору известно, такое утверждение сильно расходится с результатами, которые в случае СН получены в других работах. Поэтому представляется важным хотя бы в предварительном порядке выяснить причину расхождения.

Как отмечено во введении, главным здесь является вопрос о марковости потенциала $V(x)$, определяемого (9), где между случайными координатами $X_{n}$ нет никакой корреляции. На первьй взгляд может показаться, что это обстоятельство указывает на марковский характер $V(x)$. В этом случае возникло бы противоречие между результатом предыдущего раздела и работой [13]. Однако на самом деле при соблюдении условий предыдущего раздела потенциал $V(x)$, определяемый (9), марковским не является. В этом можно убедиться, если учесть, что, для того чтобы потенциал был марковским, необходимо сушествование такого малого интервала $x_{0}$ (порядка не более среднего расстояния между соседними координатами $X_{n}$ ), что при любых $\Delta x \geq x_{0}$

$$
\langle V(x) V(x+\Delta x)\rangle=0 .
$$

В (67) угловые скобки обозначают усреднение. В приложении показано, что при соблюдении условия равномерной сходимости (37) относительно $X_{n}$ и абсолютной интегрируемости $U(x)$

$$
\langle V(x) V(x+\Delta x)\rangle=N \int_{-\infty}^{\infty} U(x) U(x+\Delta x) d x+N^{2}\left(\int_{-\infty}^{\infty} U(x) d x\right)^{2} .
$$

Нетрудно видеть, что в обшем случае уравнение (68) условию (67) не удовлетворяет.

\section{ПРИЛОЖЕНИЕ}

Согласно (9), (37)

$$
\begin{aligned}
\langle V(x) V(x+\Delta x)\rangle= & \left\langle\sum_{m} \sum_{n} U\left(x-X_{m}\right) U\left(x-X_{n}+\Delta x\right)\right\rangle= \\
= & \frac{1}{4 \pi^{2}}\left\langle\sum_{m} \sum_{n} \int_{-\infty}^{\infty} d q \int_{-\infty}^{\infty} d q^{\prime} R(q) R\left(q^{\prime}\right) \times\right. \\
& \left.\times e^{i\left(q+q^{\prime}\right) x} e^{-i\left(q X_{m}+q^{\prime} X_{n}\right)} e^{i q^{\prime} \Delta x}\right\rangle .
\end{aligned}
$$


В условиях равномерной сходимости интегралов по $q$ и $q^{\prime}$ относительно $X_{n}$ и $X_{m}$ операции интегрирования и суммирования с усреднением можно поменять местами. В результате получим

$$
\begin{aligned}
\langle V(x) V(x+\Delta x)\rangle= & \frac{1}{4 \pi^{2}} \int_{-\infty}^{\infty} d q \int_{-\infty}^{\infty} d q^{\prime} R(q) R\left(q^{\prime}\right) \times \\
& \times e^{i\left(q+q^{\prime}\right) x} e^{i q^{\prime} \Delta x}\left\langle\sum_{m} \sum_{n} e^{-i\left(q X_{m}+q^{\prime} X_{n}\right)}\right\rangle .
\end{aligned}
$$

В условиях, когда корреляция между координатами $X_{n}$ отсутствует [22]

$$
\left\langle\sum_{m} \sum_{n} e^{-i\left(q X_{m}+q^{\prime} X_{n}\right)}\right\rangle=N Z \delta_{q,-q^{\prime}}+N Z(N Z-1) \delta_{q, 0} \delta_{q^{\prime}, 0},
$$

где $Z$ - длина образца. Если $Z \rightarrow \infty$, то

$$
\frac{Z}{2 \pi} \delta_{q,-q^{\prime}} \rightarrow \delta\left(q+q^{\prime}\right), \quad \frac{Z}{2 \pi} \delta_{q, 0} \rightarrow \delta(q)
$$

Поэтому, подставив (П.3), (П.4) в (П.2), будем иметь

$$
\begin{aligned}
\langle V(x) V(x+\Delta x)\rangle= & \frac{N}{2 \pi} \int_{-\infty}^{\infty} d q \int_{-\infty}^{\infty} d q^{\prime} R(q) R\left(q^{\prime}\right) e^{i\left(q+q^{\prime}\right) x} e^{i q^{\prime} \Delta x} \delta\left(q+q^{\prime}\right)+ \\
& +N^{2} \int_{-\infty}^{\infty} d q R(q) \delta(q) e^{i q x} \int_{-\infty}^{\infty} d q^{\prime} R\left(q^{\prime}\right) \delta\left(q^{\prime}\right) e^{i q^{\prime}(x+\Delta x)}= \\
= & \frac{N}{2 \pi} \int_{-\infty}^{\infty} d q R(q) R(-q) e^{-i q \Delta x}+N^{2}(R(0))^{2}
\end{aligned}
$$

Ho

$$
R( \pm q)=\int_{-\infty}^{\infty} U(x) e^{\mp i q x} d x
$$

Отсюда

$$
\begin{aligned}
\frac{N}{2 \pi} \int_{-\infty}^{\infty} d q R(q) R(-q) e^{i q \Delta x} & =\frac{N}{2 \pi} \int_{-\infty}^{\infty} d x \int_{-\infty}^{\infty} d x^{\prime} \int_{-\infty}^{\infty} d q e^{-i\left(x-x^{\prime}+\Delta x\right) q} U(x) U\left(x^{\prime}\right)= \\
& =N \int_{-\infty}^{\infty} d x \int_{-\infty}^{\infty} d x^{\prime} U(x) U\left(x^{\prime}\right) \delta\left(x-x^{\prime}+\Delta x\right)= \\
& =N \int_{-\infty}^{\infty} d x U(x) U(x+\Delta x), \\
R(0) & =\int_{-\infty}^{\infty} U(x) d x .
\end{aligned}
$$

Подставив (П.7), (П.8) в (П.5), получим (68).

\section{Список литературы}

[1] Mott N.F., Twose W. D. // Adv. Phys. 1961. V. 10. № 38. P. 107-163.

[2] Borland R. E. // Proc. Roy. Soc. (London) 1963. V. A529. P. 274-545. 
[3] Ishii K. // Progr. Theor. Phys. (Suppl.) 1973. V. 53. P. 77-138.

[4] Delyon F., Kunz H., Souillard B. // J. Phys. A. 1983. V. 16. № 1. P. 25-42.

[5] Березинский В. Л. // ЖЭТФ. 1973. Т. 65. № 3. С. 1251-1266.

[6] Halperin B. I. // Adv. Chem. Phys. 1967. V. 8. P. 123-177.

[7] Theodorou G., Cohen M. H. // Phys. Rev. B. 1976. V. 13. №10. P. 4597-4606.

[8] Azbel M. Ya. // Sol. State Commun. 1981. V. 37. № 10. P. 789-790.

[9] Denbigh J.S., Rivier N. // J. Phys. C. 1979. V. 12. № 3. P. L107-L110.

[10] Brezini A. // Phys. Stat. Sol. (b). 1985. V. 128. № 1. P. K81-K84.

[11] Bloch A. N., Weisman R. B., Varma C. M. // Phys. Rev. Lett. 1972. V. 28. № 12. P. 753-756.

[12] Бонч-Бруевич В. Л., Звягин И. П., Кайпер Р., Миронов А.Г., Эндерлайн Р., Эссер Б. Электронная теория неупорядоченных полупроводников. М.: Наука, 1981.

[13] Лифиич И. М., Гредескул С. А., Пастур Л. А. Введение в теорию неупорядоченных систем. М.: Наука, 1982.

[14] Гинзбург Л. П. // ФТТ. 1992. Т. 34. № 1. С. 164-167.

[15] Молчанов А. М. // Труды Московского математического общества. 1953. Т. 2. С. 169-198.

[16] Roberts G. O. // Ann. of Prob. 1991. V. 19. № 4. P. 1689-1731.

[17] Brezini A., Sebbani M., Behill F. // Phys. Stat. Sol.(b). 1986. V. 138. P. K137-K142.

[18] Бычков Ю. А. // Письма в ЖЭТФ. 1973. Т. 17. № 5. С. 266-267.

[19] Костадинов И. З. // Письма в ЖЭТФ. 1975. Т. 21. № 2. С. 105-107.

[20] Kaveh M., Mott N. F. // J. Phys. C. 1983. V. 16. № 29. P. L1067-L1072.

[21] Тер-Хаар Д. Введение в физику систем многих частиц. М.: ИЛ, 1961.

[22] Dewel G. // Physica. 1969. V. 44. №1. P. 120-142.

Московский технический университет

связи и информатики

Поступила в редакцию 2.III.1995 г.

\section{P. Ginzburg \\ THE PROBLEM OF LOCALIZATION IN ONE-DIMENSIONAL DISORDERED SYSTEMS (A NEW APPROACH)}

The theorem proved in 1951 by Molchanov [15] is utilized to investigate the problem of localization of one-electron states in one-dimensional disordered systems. The theorem permits to treat the problem in a general way and establishes a new criterion of localization, which is based on the asymptotic features of a random potential. It is shown that in the case of diagonal disorder the theorem does not lead to new results; namely, all the states are found to be localized. However, in the case of structural disorder it follows from the theorem that all the states can be delocalized under relatively weak restrictions. 